PROCEEDINGS OF THE

AMERICAN MATHEMATICAL SOCIETY

Volume 133, Number 2, Pages 577-585

S 0002-9939(04)07537-9

Article electronically published on August 25, 2004

\title{
ON LIFTING PROPERTIES FOR CONFLUENT MAPPINGS
}

\author{
JANUSZ J. CHARATONIK AND JANUSZ R. PRAJS
}

(Communicated by Alan Dow)

\begin{abstract}
Known results about lifting of paths for covering, light open and light confluent mappings are in some sense extended for all confluent mappings with the domain being a continuum having the arc property of Kelley. As an application we prove that each confluently tree-like continuum has the fixed point property.
\end{abstract}

\section{INTRODUCTION}

Classical theorems about lifting paths for covering, branched covering and related mappings have been generalized to much larger classes of light open, light confluent or light locally confluent mappings; see [2, [6], [16], [12] and the references therein. These generalizations were initiated by the result of G. T. Whyburn about lifting arcs and dendrites for light open mappings, [20, Theorem 2.4, p. 188]. A natural question that appears is whether we can get any further results in this direction so that the assumption of lightness for these mappings can be relaxed. In general, the answer is negative, even in the case when the mapping is both open and monotone; see Remarks 2.2 below.

In many applications, however, liftings can be replaced by approximative liftings, i.e., mappings for which the corresponding diagrams commute up to an (arbitrarily small) $\varepsilon>0$. The main goal of this paper is to extend the lifting theorems to the class of all confluent mappings with the compact domains having the arc property of Kelley so that the resulting mapping is an approximative lifting. The arc property of Kelley is a natural property shared by all locally connected compacta and some other classes of spaces such as, for example, continua admitting, for each $\varepsilon>0$, confluent $\varepsilon$-mapping onto a locally connected continuum, or absolute retracts for many natural classes of hereditarily unicoherent and tree-like continua; see [4] and [5].

We present an application of these results by showing that confluently tree-like continua have the fixed point property. For other related results see [2], 6], [8], [12] [16], [19].

By a space we mean a metric space, and a mapping means a continuous function. Let an $\varepsilon>0$ be given. A mapping $f: X \rightarrow Y$ from a continuum $X$ is called an $\varepsilon$-mapping provided that diam $f^{-1}(f(x))<\varepsilon$ for each point $x \in X$. For a mapping

Received by the editors July 9, 2001 and, in revised form, January 15, 2003.

2000 Mathematics Subject Classification. Primary 54C25, 54E40, 54F15, 54F50.

Key words and phrases. Arc property of Kelley, confluent mapping, continuum, lifting, locally connected, tree-like. 
$f: A \rightarrow B$, where $A$ and $B$ are subspaces of a space $X$ with a metric $d$, we define $d(f)=\sup \{d(x, f(x)): x \in A\}$. For any nonempty subset $A$ of a space $X$ and any $\varepsilon>0$ we denote by $N_{X}(A, \varepsilon)$ the open $\varepsilon$-neighborhood of $A$ in $X$. The symbol $\mathbb{N}$ stands for the set of all positive integers. Ls $A_{n}$ denotes the limit superior of a sequence of sets $A_{n}$, respectively, as defined for example in [17, 4.9, p. 56].

A tree means a graph containing no simple closed curve. A tree $T$ is said to have the order at most $n$ provided that each point of $T$ has its order (i.e., the number of maximal free arcs emanating from the point) not greater than $n$. A continuum is said to be tree-like provided that for each $\varepsilon>0$ it admits an $\varepsilon$-mapping onto a tree. A dendrite means a locally connected continuum containing no simple closed curve. A continuum that is hereditarily unicoherent (i.e., the intersection of any two of its subcontinua is connected) and arcwise connected is called a dendroid.

A mapping $f: X \rightarrow Y$ between continua is said to be confluent provided that for each subcontinuum $Q$ of $Y$ and for each component $K$ of $f^{-1}(Q)$ the equality $f(K)=Q$ holds.

Obviously each monotone mapping is confluent, and also each open one is; see [20, Theorem 7.5, p. 148]. For relations between these classes of mappings and for their properties, see e.g. [13].

A path in a space $X$ means a mapping $p:[0,1] \rightarrow X$.

Given a compact space $X$ and a space $Y$ equipped with a metric $d_{Y}$, we denote by $\rho$ the supremum metric in the space of all mappings between $X$ and $Y$, i.e., $\rho(f, g)=\sup \left\{d_{Y}(f(x), g(x)): x \in X\right\}$ for any two mappings $f, g: X \rightarrow Y$.

A continuum $X$ is said to have the property of Kelley provided that for each point $a \in X$, for each subcontinuum $K$ of $X$ containing $a$ and for each sequence of points $a_{n}$ converging to $a$ there exists a sequence of subcontinua $K_{n}$ of $X$ containing $a_{n}$ and converging to the continuum $K$. The property, introduced by J. L. Kelley in [11, p. 26], has been recognized as an important tool in investigation of various properties of continua, and is interesting in its own right.

Investigating absolute retracts for some classes of continua, we have found that the following concept of the arc property of Kelley turns out to be both natural and useful. See [4, Definition 3.3].

A continuum $X$ is said to have the arc property of Kelley provided that for each point $a \in X$, for each subcontinuum $K$ of $X$ containing $a$ and for each sequence of points $a_{n} \in X$ converging to $a$ there exists a sequence of arcwise connected (equivalently: locally connected) subcontinua $K_{n}$ of $X$ containing $a_{n}$ and converging to the continuum $K$. The following equivalent form of the arc property of Kelley will be used; see [5] Statement 2.16].

Statement 1.1. A continuum $X$ with a metric $d$ has the arc property of Kelley if and only if for each $\varepsilon>0$ there is an $\eta=\eta(\varepsilon)>0$ such that for each subcontinuum $K$ of $X$ and for all points $p, q \in K$ and $r \in X$ with $d(p, r)<\eta$, there is an arc $A \subset X$ from $r$ to some point $s$ such that $A \subset N_{X}(K, \varepsilon)$ and $d(q, s)<\varepsilon$.

\section{LIFTINGS FOR CONFLUENT MAPPINGS}

All original results of the paper are presented in this section. Namely, we generalize lifting theorems presented in [20 Theorem 2.4, p. 188] as well as in [8, Theorem 2, p. 574], [12] and [15, Theorem 1, p. 41] so that light open, light confluent and light locally confluent mappings are replaced by any (not necessarily light) confluent ones. The obtained mappings will be approximative liftings in this 
generalization (compare Remark 2.2). We begin with the case when the domain of the lifted mapping is an arc.

We will use the real function $\eta: \mathbb{R}_{+} \rightarrow \mathbb{R}_{+}$as defined in Statement 1.1 such that $\eta(\varepsilon)<\varepsilon$, with the convention $\eta^{0}(\varepsilon)=\varepsilon$ and $\eta^{n+1}(\varepsilon)=\eta\left(\eta^{n}(\varepsilon)\right)$ for each $n \in \mathbb{N}$.

Theorem 2.1. Let $X$ and $Y$ be continua such that $X$ has the arc property of Kelley. Let $f: X \rightarrow Y$ be a confluent surjection, and $p:[0,1] \rightarrow Y$ be a path. Then for each $\varepsilon>0$ there is a $\delta>0$ such that for each point $a \in N_{X}\left(f^{-1}(p(0)), \delta\right)$ there is a path $\tilde{p}:[0,1] \rightarrow X$ satisfying $\tilde{p}(0)=a$ and $\rho(f \circ \tilde{p}, p)<\varepsilon$, i.e., the following diagram $\varepsilon$-commutes:

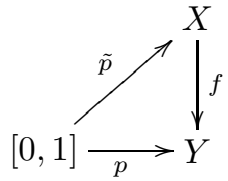

Moreover, if $p$ is an embedding, then $\tilde{p}$ can also be an embedding.

Proof. Let $d_{X}$ and $d_{Y}$ be metrics in $X$ and $Y$, respectively. Divide [0,1] into $n$ subsegments by points $0=t_{0}<t_{1}<\cdots<t_{n}=1$ such that $\operatorname{diam} p\left(\left[t_{i-1}, t_{i}\right]\right)<\frac{\varepsilon}{2}$ for each $i \in\{1, \ldots, n\}$. Let $\delta_{1}=\delta_{1}\left(\frac{\varepsilon}{2}\right)$ be as in the definition of the uniform continuity of $f$, and put $\delta=\eta^{n+1}\left(\delta_{1}\right)$.

Since $a \in N_{X}\left(f^{-1}(p(0)), \delta\right)$, there is a point $q_{0} \in f^{-1}(p(0))$ such that $d_{X}\left(a, q_{0}\right)<$ $\delta$. Denote by $C_{1}$ the component of $f^{-1}\left(p\left(\left[t_{0}, t_{1}\right]\right)\right)$ that contains the point $q_{0}$. Since $f$ is confluent, we have $f\left(C_{1}\right)=p\left(\left[t_{0}, t_{1}\right]\right)$. So there is a point $q_{1} \in C_{1}$ such that $f\left(q_{1}\right)=p\left(t_{1}\right)$. By Statement 1.1 there is an arc $A_{1} \subset X$ from $a_{0}=a$ to a point $a_{1}$ such that $A_{1} \subset N_{X}\left(C_{1}, \eta^{n}(\delta)\right)$ and $\left.d_{X}\left(a_{1}, q_{1}\right)<\eta^{n}(\delta)\right)$. Let $C_{2}$ be the component of $f^{-1}\left(p\left(\left[t_{1}, t_{2}\right]\right)\right)$ containing the point $q_{1}$, and let $q_{2} \in C_{2} \cap f^{-1}\left(p\left(t_{2}\right)\right)$. Again there is an arc $A_{2} \subset X$ from $a_{1}$ to $a_{2}$ such that $A_{2} \subset N_{X}\left(C_{2}, \eta^{n-1}(\delta)\right)$ and $d_{X}\left(a_{2}, q_{2}\right)<\eta^{n-1}(\delta)$. In general, if $C_{i}$ is the component of $f^{-1}\left(p\left(\left[t_{i-1}, t_{i}\right]\right)\right)$ containing the point $q_{i-1}$ and if $q_{i} \in C_{i} \cap f^{-1}\left(p\left(t_{i}\right)\right)$, we may define arcs $A_{i} \subset X$ from $a_{i-1}$ to $a_{i}$ such that $A_{i} \subset N_{X}\left(C_{i}, \eta^{n-i+1}(\delta)\right)$ and $d_{X}\left(a_{i}, q_{i}\right)<\eta^{n-i+1}(\delta)$.

In the general case of the theorem, we let $\tilde{p} \mid\left[t_{i-1}, t_{i}\right]:\left[t_{i-1}, t_{i}\right] \rightarrow A_{i}$ be a homeomorphism such that $\tilde{p}\left(t_{i}\right)=a_{i}$. In the case when $p$ is an embedding, the construction can be done in such a way (the details are left to the reader) that the arcs $A_{1}, \ldots, A_{n}$ form a chain, i.e., $A_{i} \cap A_{j} \neq \emptyset$ if and only if $|i-j| \leq 1$. Finally, let $A$ be an arc contained in $A_{1} \cup \cdots \cup A_{n}$ from $a_{0}$ to $a_{n}$. Put $a_{0}^{\prime}=a_{0}, a_{n}^{\prime}=a_{n}$, and for each $i \in\{1, \ldots, n-1\}$ choose a point $a_{i}^{\prime} \in A \cap A_{i} \cap A_{i+1}$. For each $i \in\{1, \ldots, n\}$ let $\tilde{p} \mid\left[t_{i-1}, t_{i}\right]:\left[t_{i-1}, t_{i}\right] \rightarrow a_{i-1}^{\prime} a_{i}^{\prime}$ be any homeomorphism onto the arc $a_{i-1}^{\prime} a_{i}^{\prime} \subset A$ such that $\tilde{p}\left(t_{i}\right)=a_{i}^{\prime}$. We will show that $\tilde{p}:[0,1] \rightarrow X$ satisfies, in both the cases, the needed conditions. Really, take a number $t \in\left[t_{i-1}, t_{i}\right]$ and choose $x \in C_{i+1}$ so that $d_{X}(\tilde{p}(t), x)<\delta=\delta\left(\frac{\varepsilon}{2}\right)$. Then, since both $p(t)$ and $f(\tilde{p}(t))$ are in the arc $p\left(\left[t_{i-1}, t_{i}\right]\right)$, we have $d_{Y}(p(t), f(\tilde{p}(t))) \leq d_{Y}(p(t), f(x))+d_{Y}(f(x), f(\tilde{p}(t)))<\frac{\varepsilon}{2}+\frac{\varepsilon}{2}=\varepsilon$. The proof is complete.

Remarks 2.2. (a) The assumption of Theorem 2.1 that the continuum $X$ has the arc property of Kelley is essential, since there exists a monotone and open mapping of the circle of pseudo-arcs, [1, p. 189], onto a circle; note that the domain of the mapping contains no arc. 
(b) The results of Theorem 2.1 cannot be improved by saying that the mappings $p$ and $f \circ \tilde{p}$ are identical. As the needed example one can take a monotone decomposition of a 2-sphere into pseudo-arcs (see [18, Theorem 8, p. 29]). According to [18, Proposition 1, p. 26] no arc in the domain is mapped onto an arc in the range, while both the domain and the range spaces are locally connected continua (thus they have the arc property of Kelley) and the mapping is not only confluent but also monotone and open simultaneously.

Theorem 2.1, in the case when $p$ is an embedding, will be used in the following equivalent form.

Statement 2.3. Let $X$ and $Y$ be continua such that $X$ has the arc property of Kelley, let $p:[0,1] \rightarrow Y$ be a path, and let $f: X \rightarrow Y$ be a confluent mapping. Then for each sequence of points $a_{n} \in X$ with $\operatorname{Ls}\left\{a_{n}\right\} \subset f^{-1}(p(0))$ there is a sequence of paths $\tilde{p}_{n}:[0,1] \rightarrow X$ such that $\lim \rho\left(f \circ \tilde{p}_{n}, p\right)=0$.

Moreover, if $p$ is an embedding, then $\tilde{p}_{n}$ can also be an embedding.

The next result is an extension of Theorem 2.1 with a tree in place of an arc.

Theorem 2.4. Let $f: X \rightarrow Y$ be a confluent mapping between continua $X$ and $Y$ such that $X$ has the arc property of Kelley, and let $g: T \rightarrow Y$ be a mapping from a tree $T$ with some point $b \in T$. Then for each sequence of points $a_{n} \in X$ such that $\operatorname{Ls}\left\{a_{n}\right\} \subset f^{-1}(g(b))$ there is a sequence of mappings $\tilde{g}_{n}: T \rightarrow X$ satisfying $\tilde{g}_{n}(b)=a_{n}$ and $\lim \rho\left(f \circ \tilde{g}_{n}, g\right)=0$, i.e., the following diagram $\varepsilon_{n}$-commutes for some sequence $\left\{\varepsilon_{n}\right\} \rightarrow 0$ :

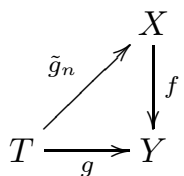

Proof. We apply induction with respect to the number $i$ of end points of $T$ distinct from $b$. If $i=1$, then $T$ is an arc and $b$ is an end point of $T$. Then we identify $T$ and $[0,1]$ and apply Theorem 2.3 for $g=p$ letting $\tilde{g}_{n}=\tilde{p}_{n}$.

Assume now that the conclusion is true for $i \in\{1, \ldots, k-1\}$, where $k>1$ is an integer. We will show that it is true for $i=k$. Let $\left\{a_{n}\right\}$ be a sequence of points in $X$ such that $\operatorname{Ls}\left\{a_{n}\right\} \subset f^{-1}(g(b))$.

If the point $b$ separates $T$ into $m$ components $C_{1}, \ldots, C_{m}$, we apply the inductive assumption to $b$ and trees $T_{j}=\operatorname{cl} C_{j}$ for $j \in\{1, \ldots, m\}$ separately, obtaining mappings $\tilde{g}_{n, j}: T_{j} \rightarrow X$. Observe that for each $n \in \mathbb{N}$ the mapping $\tilde{g}_{n}: T \rightarrow X$ defined as the combination of the mappings $\tilde{g}_{n, j}$, i.e., $\tilde{g}_{n} \mid T_{j}=\tilde{g}_{n, j}$ for each $j$, is well defined because $\tilde{g}_{n, j}(b)=a_{n}$ for each $j \in\{1, \ldots, m\}$. Then $\tilde{g}_{n}$ are the needed mappings.

If $b$ is an end point of $T$, then there is a ramification point $c \in T \backslash\{b\}$. Let $D_{1}$ be the closure of the component of $T \backslash\{c\}$ that contains $b$, and $D_{2}, \ldots, D_{m}$ be the closures of other components of $T \backslash\{c\}$. First we apply the inductive assumption to $b$ and the tree $D_{1}$ obtaining, for each $n \in \mathbb{N}$, mappings $\tilde{g}_{n, 1}: D_{1} \rightarrow X$. Next the inductive assumption is applied to the trees $D_{2}, \ldots, D_{m}$ with the distinguished point $c$ (in place of $b$ ), and the sequence $\tilde{a}_{n}=\tilde{g}_{n, 1}(c)$ in place of $a_{n}$. We see that $\operatorname{Ls}\left\{\tilde{a}_{n}\right\} \subset f^{-1}(g(c))$. Thus for each $j \in\{1, \ldots, m\}$ there are mappings $\tilde{g}_{n, j}: D_{j} \rightarrow$ $X$ such that the $f \circ \tilde{g}_{n, j}: D_{j} \rightarrow Y$ converge to $g \mid D_{j}$. The mappings $\tilde{g}_{n}$ that are combinations of mappings $\tilde{g}_{n, j}$ for $j \in\{1, \ldots, m\}$ satisfy the conclusion. 
Theorem 2.5. Under the conditions of Theorem 2.4, if, additionally, $g$ is an embedding, the order of $T$ is less than or equal to 3 and $b$ is an end point of $T$, then the mappings $\tilde{g}_{n}: T \rightarrow X$ in the conclusion of Theorem 2.4 can be chosen to be embeddings.

Proof. We apply notation from the proof of Theorem 2.4, and again proceed by induction. For the first inductive step, i.e., for $i=1$, identifying $T$ with $[0,1], b$ with 0 and $g$ with $p$, we see that $\tilde{g}_{n}=\tilde{p}_{n}$ can be embeddings by Statement 2.3. Assume that the conclusion is true for $i \in\{1, \ldots, k-1\}$, where $k>1$ is an integer. We will show that it is true for $i=k$. Let again $\left\{a_{n}\right\}$ be a sequence of points in $X$ such that $\operatorname{Ls}\left\{a_{n}\right\} \subset f^{-1}(g(b))$.

Since $b$ is an end point of $T$, there is a ramification point $c \in T \backslash\{b\}$. Let $D_{1}$ be the closure of the component of $T \backslash\{c\}$ that contains $b$, and let $D_{2}, D_{3}$ be the closures of other components of $T \backslash\{c\}$. Arguing as in the proof of Theorem 2.4, by the inductive assumption, we obtain embeddings $\hat{g}_{n, 1}: D_{1} \rightarrow X$ with $\hat{g}_{n, 1}(b)=a_{n}$ and embeddings $\hat{g}_{n, 2}: D_{2} \rightarrow X$ as well as $\hat{g}_{n, 3}: D_{3} \rightarrow X$ such that their combinations, $\hat{g}_{n}: T \rightarrow X$, have the property $\lim \left(f \circ \hat{g}_{n}\right)=g$.

Now we will slightly modify the mappings $\hat{g}_{n}$ in small neighborhoods of $c$ to obtain embeddings $\tilde{g}_{n}$. Since, for $j \in\{1,2,3\}$, the mappings $\hat{g}_{n, j}$ are embeddings with the compositions $f \circ \hat{g}_{n, j}$ converging to $g$ on $D_{j}$, there is a sequence of open neighborhoods $U_{n}$ of $g(c)$ in $Y$ such that, for each $n \in \mathbb{N}$,

(2.5.1) there is a simple triod $T_{n} \subset T \cap U_{n}$ having $c$ as its vertex and such that $\hat{g}_{n} \mid \operatorname{cl}\left(T \backslash T_{n}\right)$ is an embedding;

(2.5.2) $\hat{g}_{n}\left(T_{n}\right) \subset f^{-1}\left(U_{n}\right)$;

(2.5.3) for each end point $e_{n}^{j}$ of $T_{n}$, where $j \in\{1,2,3\}$ and $e_{n}^{j} \in D_{j}$, we have $\operatorname{ord}\left(e_{n}^{j}, T\right)=\operatorname{ord}\left(\hat{g}_{n}\left(e_{n}^{j}\right), \hat{g}_{n}(T)\right)=2$;

(2.5.4) $\lim \operatorname{diam} U_{n}=0$.

Fix an arbitrary $n \in \mathbb{N}$. Let $e_{n}^{1} e_{n}^{2}$ be an arc in $T_{n}$, and let $A_{n}$ be an arc in $\hat{g}_{n}\left(e_{n}^{1} e_{n}^{2}\right)$ irreducible between $\hat{g}_{n}\left(e_{n}^{1}\right)$ and $\hat{g}_{n}\left(e_{n}^{2}\right)$. Furthermore, let $B_{n}$ be an arc in $\hat{g}_{n}\left(e_{n}^{1} e_{n}^{3}\right)$ irreducible between $\hat{g}_{n}\left(e_{n}^{3}\right)$ and $A_{n}$. The union $A_{n} \cup B_{n}$ is a simple triod in $f^{-1}\left(U_{n}\right)$ with end points $\hat{g}_{n}\left(e_{n}^{j}\right)$ for $j \in\{1,2,3\}$. We extend the embedding $\hat{g}_{n} \mid \operatorname{cl}\left(T \backslash T_{n}\right)$ to a mapping $\tilde{g}_{n}: T \rightarrow X$ letting $\tilde{g}_{n} \mid T_{n}$ be any homeomorphism from $T_{n}$ onto $A_{n} \cup B_{n}$ such that $\tilde{g}_{n}\left(e_{n}^{j}\right)=\hat{g}_{n}\left(e_{n}^{j}\right)$ for $j \in\{1,2,3\}$. Observe that the $\tilde{g}_{n}$ so defined is an embedding. Since $\lim \operatorname{diam} U_{n}=0$, we have $\lim \rho\left(f \circ \hat{g}_{n}, f \circ \tilde{g}_{n}\right)=0$. Thus $\lim \rho\left(f \circ \tilde{g}_{n}, g\right)=0$, as needed. The proof is complete.

Remarks 2.6. (a) The assumption of Theorem 2.5 that each point of $T$ has order in $T$ at most 3 is essential. Indeed, let $X$ be a linear graph of the shape of the letter $\mathrm{H}$, and let a monotone mapping $f: X \rightarrow Y$ shrink the horizontal bar of $\mathrm{H}$ to a point. Then $Y$ is a 4-od of the shape of the letter $\mathrm{X}$ and there is no homeomorphic copy of $Y$ in $X$.

(b) An example can be constructed showing that the assumption of Theorem 2.5 on $T$ being a tree cannot be relaxed to being a dendrite (of order at most 3 ).

Recall that a compact space $X$ is called an approximative absolute retract (written AAR) provided that whenever $X$ is embedded in a compact space (or, equivalently, in the Hilbert cube) $Y$, for each $\varepsilon>0$ there is a mapping $f: Y \rightarrow X$ such that $d(f \mid X)<\varepsilon$.

An AAR continuum $X$ is called a pointed AAR if, for any fixed point $x_{0} \in X$, the mapping $f: Y \rightarrow X$ required by the above definition of an AAR additionally 
satisfies $f\left(x_{0}\right)=x_{0}$. One can use the example of the $\sin (1 / x)$-curve to see that the class of AAR tree-like continua is essentially larger than the class of pointed ones.

Observation 2.7. A tree-like continuum $X$ is a (pointed) AAR if and only if (for each $x_{0} \in X$ and) for each $\varepsilon>0$ there exists a mapping $f: X \rightarrow T$ with $d(f)<\varepsilon$ onto a tree $T$ contained in $X$ (such that $\left.f\left(x_{0}\right)=x_{0}\right)$.

Proof. If $X$, embedded in a space $Y$, admits such mappings $f$, then $f$ can be extended to $Y$ because $T$ is an AR. On the other hand, if a tree-like (pointed) AAR $X$ is embedded in the Hilbert cube $\mathbb{Q}$, then for each $\varepsilon>0$ there is a mapping $f^{\prime}: \mathbb{Q} \rightarrow X$ such that $f^{\prime} \mid X$ is an $\varepsilon$-mapping (and $f^{\prime}\left(x_{0}\right)=x_{0}$ ). Thus $f^{\prime}(X)$ is a locally connected tree-like continuum, i.e., a dendrite. The mapping $f^{\prime} \mid X$ can be slightly modified to an $\varepsilon$-mapping $f: X \rightarrow X$ such that $f(X)$ is a tree (and $\left.f\left(x_{0}\right)=x_{0}\right)$.

Theorem 2.8. Let $f: X \rightarrow Y$ be a confluent mapping between continua $X$ and $Y$ such that $X$ has the arc property of Kelley, and let $g: Z \rightarrow Y$ be a mapping from a tree-like $A A R$ continuum $Z$. Then for each $\varepsilon>0$ there is a mapping $\tilde{g}: Z \rightarrow X$ such that $\rho(f \circ \tilde{g}, g)<\varepsilon$, i.e., the following diagram $\varepsilon$-commutes:

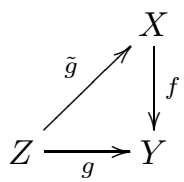

Moreover, if $Z$ is a pointed $A A R$ tree-like continuum, then for each point $z \in Z$ there exists a $\delta>0$ such that for each $a \in N_{X}\left(f^{-1}(g(z))\right.$, $\left.\delta\right)$ we can additionally obtain $\tilde{g}(z)=a$.

Proof. By Observation 2.7 there are mappings $k_{n}: Z \rightarrow T_{n}$ onto trees $T_{n} \subset Z$ such that $\lim \rho\left(k_{n}\right.$, id $\left.\mid Z\right)=0$. By Theorem 2.4 applied for $g \mid T_{n}: T_{n} \rightarrow Y$ there are mappings $\tilde{g}_{n}: T_{n} \rightarrow X$ such that $\rho\left(f \circ \tilde{g}_{n}, g \mid T_{n}\right)<\frac{1}{n}$. Thus

$$
\lim \rho\left(f \circ \tilde{g}_{n} \circ k_{n},\left(g \mid T_{n}\right) \circ k_{n}\right)=0 \quad \text { and } \quad \lim \rho\left(\left(g \mid T_{n}\right) \circ k_{n}, g\right)=0 .
$$

Therefore $\lim \rho\left(f \circ\left(\tilde{g}_{n} \circ k_{n}\right), g\right)=0$, and we choose $\tilde{g}=\tilde{g}_{n} \circ k_{n}$ for sufficiently large $n$, which completes the proof of the general case.

To show the pointed case, fix a point $z \in Z$. Let $k_{n}: Z \rightarrow T_{n}$ be as in the previous case. Since $Z$ is a pointed AAR we can additionally assume that $k_{n}(z)=z$. Suppose that there is no required $\delta$. Thus there is a sequence $\left\{a_{m}\right\} \subset X$ such that $\operatorname{Ls}\left\{a_{m}\right\} \subset f^{-1}(g(z))$ and such that for each $m$ there is no required $\tilde{g}$ with $\tilde{g}(z)=a_{m}$. By Theorem 2.4 applied again for $g \mid T_{n}: T_{n} \rightarrow Y$ there are mappings $\tilde{g}_{n, m}: T_{n} \rightarrow X$ such that for each $n \in \mathbb{N}$ we have $\lim _{m} \rho\left(f \circ \tilde{g}_{n, m}, g \mid T_{n}\right)=0$ and $\tilde{g}_{n, m}(z)=a_{m}$. Using the "diagonal procedure" we can find a sequence of indices $m_{n}$ such that $\lim _{n} \rho\left(f \circ\left(\tilde{g}_{n, m_{n}} \circ k_{n}\right), g \mid T_{n}\right)=0$. Thus, for sufficiently large $n$, for $a=a_{n}$ and $\tilde{g}=\tilde{g}_{n, m_{n}} \circ k_{n}$, we have $\tilde{g}(z)=a$ and $\rho(f \circ \tilde{g}, g)<\varepsilon$, a contradiction.

To see some applications of Theorem 2.8 we recall the needed concepts.

A compact $X$ is called an absolute retract for a class $\mathcal{T} \mathcal{L}$ of tree-like continua (written $\operatorname{AR}(\mathcal{T} \mathcal{L})$ ) provided that whenever $X$ is embedded in a tree-like continuum $Y$, the embedded copy of $X$ is a retract of $Y$.

A subcontinuum $X$ of a continuum $Y$ is said to be terminal in $Y$ if each subcontinuum of $Y$ that intersects $X$ and $Y \backslash X$ contains $X$. A continuum $X$ is said to 
be an absolute terminal retract provided that if $X$ is embedded in a continuum $Y$ in such a way that the embedded copy, $X^{\prime}$, is a terminal subcontinuum of $Y$, then $X^{\prime}$ is a retract of $Y$ (see [7]).

A continuum $X$ is said to be confluently tree-like provided that for each $\varepsilon>0$ there is a confluent $\varepsilon$-mapping from $X$ onto a tree.

Remark 2.9. The following classes of tree-like continua are composed of AARs, and thus their members can be taken as the space $Z$ in Theorem 2.8:

(i) general case:

(2.9.1) tree-like absolute terminal retracts; this class actually is identical with treelike AARs (see [7]);

(ii) pointed case:

(2.9.2) trees;

(2.9.3) dendrites;

(2.9.3) $\operatorname{AR}(\mathcal{T} \mathcal{L})$

(2.9.4) confluently tree-like continua (see Theorem 2.12 below).

In particular, the following continua belong both to $\operatorname{AR}(\mathcal{T} \mathcal{L})$ and to the class of confluently tree-like ones: each cone over a compact 0-dimensional set (see 14 p. 183]), Knaster type continua (see [3, Corollary 3.7]), and any inverse limit of trees with confluent bonding mappings (see [3, Theorem 3.6]).

In view of Theorem 2.8 the following question seems to be of a special interest.

Question 2.10. Is each dendroid an AAR?

The above question is related to the next one about the existence of small retractions on dendroids (see [7, Questions 5.5-5.8]).

Question 2.11. Let $X$ be a dendroid. Do there exist, for each $\varepsilon>0$, a tree $T \subset X$ and an $\varepsilon$-mapping $r: X \rightarrow T$ such that $r$ is a retraction?

Question 2.11, asked by B. Knaster some 40 years ago, is still open, despite numerous attempts to solve it.

As another application of Theorem 2.8 we will prove that each confluently treelike continuum has the fixed point property. The study of the fixed point property for tree-like continua has been an exciting and challenging area in topology for many decades; see, e.g., [10] and the references therein.

Theorem 2.12. Each confluently tree-like continuum $X$ is a pointed AAR.

Proof. We will show the equivalent condition from Observation 2.7.

By Corollary 2.26 of $[5$ the continuum $X$ has the arc property of Kelley. Fix $x_{0} \in X$ and for each $n \in \mathbb{N}$ let $f_{n}: X \rightarrow T_{n}$ be confluent $\frac{1}{n}$-mappings, where $T_{n}$ are trees. Without loss of generality, we can assume that $T_{n}$ and $X$ are subsets of a common space $Y$ such that $\lim d\left(f_{n}\right)=0$. Fix $\varepsilon>0$ and choose $m \in \mathbb{N}$ so that $d\left(f_{m}\right)<\frac{\varepsilon}{2}$. Let $g_{n}^{m}: T_{m} \rightarrow X$ be approximate liftings of the identities on the trees $T_{m}$ guaranteed by Theorem 2.4, where $b=f_{n}\left(x_{0}\right)$ and $a_{n}=x_{0}$. Note that $\left(g_{n}^{m} \circ f_{n}\right)\left(x_{0}\right)=x_{0}$. For each $x \in T_{m}$ we have

$$
d\left(x, g_{n}^{m}(x)\right) \leq d\left(g_{n}^{m}(x), f_{m}\left(g_{n}^{m}(x)\right)\right)+d\left(f_{m}\left(g_{n}^{m}(x)\right), x\right) \leq d\left(f_{m}\right)+d\left(f_{m} \circ g_{n}^{m}\right),
$$

whence $d\left(g_{n}^{m}\right) \leq d\left(f_{m}\right)+d\left(f_{m} \circ g_{n}^{m}\right)$. Therefore, since $d\left(f_{m} \circ g_{n}^{m}\right) \rightarrow 0$ as $n \rightarrow \infty$, there is an $n \in \mathbb{N}$ such that $d\left(g_{n}^{m}\right)<\frac{\varepsilon}{2}$. Define $f^{\prime}=g_{n}^{m} \circ f_{m}$. Then $d\left(f^{\prime}\right) \leq$ 
$d\left(g_{n}^{m}\right)+d\left(f_{m}\right)<\frac{\varepsilon}{2}+\frac{\varepsilon}{2}=\varepsilon$, and the set $f^{\prime}(X)$ is a continuum contained in a tree-like locally connected continuum $g_{n}^{m}\left(T_{m}\right)$. Thus $f^{\prime}(X)$ is a dendrite. We can slightly modify the mapping $f^{\prime}$ to a mapping $f: X \rightarrow X$ so that $d(f)<\varepsilon, f(X)$ is a tree in $f^{\prime}(X)$ and $f\left(x_{0}\right)=x_{0}$. The proof is complete.

As a consequence of Observation 2.7 and Theorem 2.12 we have the following results.

Corollary 2.13. Each confluently tree-like continuum $X$ has the fixed point property.

Proof. Let a mapping $g: X \rightarrow X$ be given, and let $f_{n}: X \rightarrow X$ be mappings guaranteed by Theorem 2.4 for the corresponding numbers $\frac{1}{n}$. The composition $f_{n} \circ g \mid f_{n}(X)$ is a mapping from the tree $f_{n}(X)$ into itself. So it has a fixed point $x_{n}$. Observe that any accumulation point of the sequence $\left\{x_{n}\right\}$ is a fixed point of $g$.

Remark 2.14. Corollary 2.13 can also be obtained using Theorem 2.12 and the corollary in [9, p. 19] saying that each AAR has the fixed point property.

Since each confluently tree-like continuum has the arc property of Kelley (see [5. Corollary 2.26]), in connection with Corollary 2.13 one can ask the following.

Question 2.15. Does the arc property of Kelley for tree-like continua imply the fixed point property?

\section{ACKNOWLEDGMENT}

The authors thank Professor W. J. Charatonik for valuable discussions they had.

\section{REFERENCES}

[1] R. H. Bing and F. B. Jones, Another homogeneous plane continuum, Trans. Amer. Math. Soc. 90 (1959), 171-192. MR 20:7251

[2] J. J. Charatonik, W. J. Charatonik and P. Krupski, Dendrites and light open mappings, Proc. Amer. Math. Soc. 128 (2000), 1839-1843. MR 2001c:54027

[3] J. J. Charatonik, W. J. Charatonik and J. R. Prajs, Hereditarily unicoherent continua and their absolute retracts, Rocky Mountain J. Math. 34 (2004), no. 1, 83-110.

[4] J. J. Charatonik, W. J. Charatonik and J. R. Prajs, Arc property of Kelley and absolute retracts for hereditarily unicoherent continua. Colloq. Math. 97 (2003), no. 1, 49-65.

[5] J. J. Charatonik, W. J. Charatonik and J. R. Prajs, Confluent mappings and the arc property of Kelley, preprint.

[6] J. J. Charatonik and P. Krupski, Dendrites and light mappings, Proc. Amer. Math. Soc. 132 (2004), 1211-1217.

[7] J. J. Charatonik and J. R. Prajs, AANR spaces and absolute retracts for tree-like continua, Czechosl. Math. J. (to appear).

[8] E. E. Floyd, Some characterizations of interior mappings, Ann. of Math. 51 (1950), 571-575. MR 11:676a

[9] A. Granas, Fixed point theorems for the approximative ANR-s, Bull. Acad. Polon. Sci. Sér. Sci. Math. Astronom. Phys. 16 (1968), 15-19. MR 37:3501

[10] C. L. Hagopian, Fixed-point problems in continuum theory, Contemp. Math. 117 (1991), 79-86. MR 92i:54033

[11] J. L. Kelley, Hyperspaces of a continuum, Trans. Amer. Math. Soc. 52 (1942), 22-36. MR 3:315b

[12] J. Krasinkiewicz, Path-lifting property for 0-dimensional confluent mappings, Bull. Polish Acad. Sci. Math. 48 (2000), 357-367. MR 2001h:54020

[13] T. Maćkowiak, Continuous mappings on continua, Dissertationes Math. (Rozprawy Mat.) 158 (1979), 1-95. MR 81a:54034 
[14] T. Maćkowiak, Retracts of hereditarily unicoherent continua, Bull. Acad. Polon. Sci. Sér. Sci. Math. 28 (1980), 177-183. MR 82g:54063

[15] T. Maćkowiak and E. D. Tymchatyn, Some classes of locally connected continua, Colloq. Math. 52 (1987), 39-52. MR 88h:54047

[16] J. Mioduszewski, Twierdzenie o selektorach funkcyj wielowartościowych na dendrytach [A theorem on the selectors of multivalued functions on dendrites], Prace Mat. 5 (1961), 73-77; (in Polish; Russian and English summaries). MR 24:A534

[17] S. B. Nadler, Jr., Continuum theory. An introduction, Monographs and Textbooks in Pure and Applied Mathematics, no. 158, Marcel Dekker, New York, 1992. MR 93m:54002

[18] J. R. Prajs, Continuous decompositions of Peano plane continua into pseudo-arcs, Fund. Math. 158 (1998), 23-40. MR 2000b:54043

[19] S. Smale, A note on open maps, Proc. Amer. Math. Soc. 8 (1957), 391-393. MR 19:158f

[20] G. T. Whyburn, Analytic topology, Amer. Math. Soc. Colloq. Publ. 28, Providence, RI, 1942 (reprinted with corrections 1971). MR 4:86b

Instituto de Matemáticas, UnAm, Circuito Exterior, Ciudad Universitaria, 04510 MÉxico, D.F., México - And - Mathematical Institute, University of WrocŁaW, PL. GrunWaldzKi 2/4, 50384, WrocŁaW, Poland

E-mail address: jjc@math.unam.mx

Department of Mathematics and Statistics, California State University Sacramento, Sacramento, California 95819-6051 - and - Institute of Mathematics, University of Opole, ul. Oleska 48, 45-052 Opole, Poland

E-mail address: prajs@csus.edu 